\title{
ON THE BOUNDARY LIMITS OF POLYHARMONIC FUNCTIONS IN A HALF SPACE
}

Dedicated to Professor Mitsuru Ozawa on the occasion of his 60th birthday

$$
\text { By Yoshiniro Mizuta }
$$

\section{Introduction and statement of result.}

Let $R^{n}$ be the $n$-dimensional Euclidean space $(n \geqq 2)$, and set

$$
R_{+}^{n}=\left\{x=\left(x^{\prime}, x_{n}\right) \in R^{n-1} \times R^{1} ; x_{n}>0\right\} .
$$

For $\xi \in \partial R_{+}^{n}, \gamma \geqq 1$ and $a>0$, define

$$
T_{\gamma}(\xi, a)=\left\{\left(x^{\prime}, x_{n}\right) \in R_{+}^{n} ;\left|\left(x^{\prime}, 0\right)-\xi\right|<a x_{n}^{1 / r}\right\} .
$$

Recently Cruzeiro [2] proved the existence of $\lim u(x)$ as $x \rightarrow \xi, x \in T_{\gamma}(\xi, a)$, for a harmonic function $u$ with gradient in $L^{n}\left(R_{+}^{n}\right)$. In this note we are concerned with polyharmonic functions in $R_{+}^{n}$, and our purpose is to give a generalization of her result to the polyharmonic case.

For a nonnegative integer $m$, denote by $\Delta^{m}$ the Laplace operator iterated $m$ times; in particular, $\Delta^{0}$ denotes the identity operator. A function $u \in C^{\infty}\left(R_{+}^{n}\right)$ is said to be polyharmonic of order $m$ in $R_{+}^{n}$ if

$$
\Delta^{m} u=0 \quad \text { on } \quad R_{+}^{n} .
$$

For $u \in C^{m}\left(R_{+}^{n}\right)$ and $x=\left(x_{1}, \cdots, x_{n}\right) \in R_{+}^{n}$, define

$$
\left|\nabla_{m} u(x)\right|=\left\{\sum_{\lambda \mid=m}\left|D^{2} u(x)\right|^{2}\right\}^{1 / 2},
$$

where $\lambda=\left(\lambda_{1}, \cdots, \lambda_{n}\right)$ denotes a multi-index with length $|\lambda|=\lambda_{1}+\cdots+\lambda_{n}$ and $D^{\lambda}=\left(\partial / \partial x_{1}\right)^{\lambda_{1} \ldots} \ldots\left(\partial / \partial x_{n}\right)^{\lambda_{n}}$.

THEOREM. Let $m$ be a positive integer and $u$ be a function which is polyharmonic of order $m+1$ in $R_{+}^{n}$ and satısfies

$$
\int_{G}\left|\nabla_{m} u(x)\right|^{p} x_{n}^{\alpha} d x<\infty, \quad p>1, \quad \alpha<m p-1,
$$

for any bounded open set $G$ in $R_{+}^{n}$. Suppose $(\alpha+1) / p$ is not a positive integer.

Received April 21, 1983 
(i) If $n-m p+\alpha>0$, then for each $\gamma>1$ there exists a set $E_{r} \subset \partial R_{+}^{n}$ such that $H_{\gamma(n-m p+\alpha)}\left(E_{\gamma}\right)=0$ and

$$
\lim _{x \rightarrow \xi, x \in T_{\gamma}(\xi, a)} u(x)
$$

exists and is finite for any $a>0$ and any $\xi \in \partial R_{+}^{n}-E_{\gamma}$.

(ii) If $n-m p+\alpha=0$, then there exists a set $E \subset \partial R_{+}^{n}$ such that $B_{n / p, p}(E)=0$ and (2) exists and is finite for any $a>0$, any $\gamma>1$ and any $\xi \in \partial R_{+}^{n}-E$.

(iii) If $n-m p+\alpha<0$, then $\lim _{x \rightarrow \xi, x \in R_{+}^{n}} u(x)$ exists and is finite for any $\xi \in \partial R_{+}^{n}$.

Here $H_{l}$ denotes the $l$-dimensional Hausdorff measure, and $B_{l, p}$ the Bessel capacity of index $(l, p)$ (see Meyers [4]). Note the following results (cf. [4]):

(a) If $H_{n-l}(E)<\infty$, then $B_{l / p, p}(E)=0$ for any $p>1$;

(b) If $B_{l / p, p}(E)=0$ for some $p>1$, then $H_{l^{\prime}}(E)=0$ for any $l^{\prime}>n-l$.

In the case where $(\alpha+1) / p$ is a positive integer, we have the next theorem.

THEOREM'. Let $u$ be a function which is polyharmonic of order $m+1$ in $R_{+}^{n}$ and satisfies (1) for any bounded open set $G$ in $R_{+}^{n}$, where $p>1$ and $(\alpha+1) / p$ is a positive integer smaller than $m$.

(i) If $n-m p+\alpha>0$, then for each $\gamma>1$ there exists a set $E_{\gamma} \subset \partial R_{+}^{n}$ such that $E_{\gamma}$ has Hausdorff dimension at most $\gamma(n-m p+\alpha)$ and (2) exists and is finite for any $a>0$ and any $\xi \in \partial R_{+}^{n}-E_{\gamma}$.

(ii) If $n-m p+\alpha=0$, then there exists a set $E \subset \partial R_{+}^{n}$ such that $E$ has Hausdorff dimension 0 and (2) exists and is finte for any $a>0$, any $\gamma>1$ and any $\xi \in \partial R_{+}^{n}-E$.

(iii) If $n-m p+\alpha<0$, then $\lim _{x \rightarrow \xi, x \in R_{+}^{n}} u(x)$ exists and is finute for any $\xi \in \partial R_{+}^{n}$.

If $\lim _{x \rightarrow \xi, x \in T_{1}^{\prime}(\xi, a)} u(x)$ exists and is finite for any $a>0$, then $u$ is said to have a nontangential limit at $\xi$. If $u$ is a function which is polyharmonic of order $m+1$ in $R_{+}^{n}$ and satisfies (1) with $p>1$ and $\alpha<m p-1$ for any bounded open set $G$ in $R_{+}^{n}$, then $u$ has a nontangential limit at any $\xi \in \partial R_{+}^{n}$ except for those in a set $E$ with $B_{m-\alpha / p, p}(E)=0$; this result is best possible as to the size of the exceptional set in the following sense: If $E \subset \partial R_{+}^{n}, B_{m-\alpha / p, p}(E)=0$ and $-1<\alpha$ $<m p-1$, then we can find a harmonic function $u$ in $R_{+}^{n}$ which satisfies (1) with $G=R_{+}^{n}$ such that $\lim _{x \rightarrow \xi, x \in R_{+}^{n}} u(x)=\infty$ for any $\xi \in E$ (see [8; Theorems 1 and 2]). Thus (ii) of the theorem gives an improvement of [8; Theorem 1], and also the best possible result as to the size of the exceptional set.

\section{Lemmas.}

First we prepare several properties of polyharmonic functions. Let $B(x, \gamma)$ denote the open ball with center at $x$ and radius $r$. For $E \subset R^{n}$, denote the closure of $E$ by $\bar{E}$. 
LEMMA 1. Let $u$ be a function which is polyharmonic of order $m+1$ in $R_{+}^{n}$. Then there exist constants $c_{\imath}$ independent of $u$ such that

$$
r^{1-n} \int_{\partial B(x, r)} \Delta u(y) d S(y)=\sum_{\imath=1}^{m} c_{i} r^{2 \imath-2} \Delta^{2} u(x)
$$

whenever $\overline{B(x, r)} \subset R_{+}^{n}$.

Proof. By a result in [9; p. 189], there exist harmonic functions $v_{i}$ in $B\left(x, r^{\prime}\right)$ such that

$$
\Delta u(y)=\sum_{\imath=1}^{m}|y-x|^{2 \imath-2} v_{\imath}(y) \quad \text { on } B\left(x, r^{\prime}\right),
$$

where $B\left(x, r^{\prime}\right) \subset R_{+}^{n}$. Then we note that $\Delta^{2} u(x)=c_{i}^{\prime} v_{i}(x)$, so that

$$
r^{1-n} \int_{\hat{o} B(x, r)} \Delta u(y) d S(y)=\sum_{i=1}^{m} c_{\imath}^{\prime \prime} r^{2 \imath-2} v_{i}(x)=\sum_{i=1}^{m} c_{i} r^{2 \imath-2} \Delta^{2} u(x)
$$

for $r$ with $0<r<r^{\prime}$. The constants $c_{\imath}^{\prime}, c_{\imath}^{\prime \prime}$ and $c_{\imath}$ depend only on $\imath$ and the dimension $n$.

LEMMA 2. Let $u$ be a function which is polyharmonic of order $m+1$ in $R_{+}^{n}$, and let $\overline{B(x, r)} \subset R_{+}^{n}$. Then for each nonnegative integer $\imath$, $i \leqq m$, there exist constants $a_{2}^{(i)}$ independent of $u, x$ and $r$ such that

$$
\Delta^{2} u(x)=r^{-n-2 \imath} \sum_{0<|\lambda| \leq m} a_{\lambda}^{(\imath)} \int_{B(x, r)}(y-x)^{\lambda} D^{\lambda} u(y) d y .
$$

Proof. In view of $[3 ;(15)]$,

$$
\Delta^{2} u(x)=\sum_{k=0}^{m-\imath} a_{k} \rho^{k} \int_{\partial B(0,1)}\left(\frac{\partial}{\partial \rho}\right)^{k} \Delta^{2} u(x+\rho \sigma) d S(\sigma)
$$

with constants $a_{k}$. We introduce a differential operator

$$
\nu=\sum_{j=1}^{n}\left(y_{\jmath}-x_{\jmath}\right) \frac{\partial}{\partial y_{\jmath}} .
$$

Letting $I$ denote the identity operator, we note that

so that

$$
\nu^{k} \Delta^{\imath}=\Delta^{\imath}(\nu-2 i I)^{k},
$$

$$
\begin{aligned}
\rho^{n-1} \Delta^{2} u(x) & =\sum_{k=0}^{m-\imath} a_{k} \int_{\partial B(x, \rho)} \nu^{k} \Delta^{2} u(y) d S(y) \\
& =\sum_{k=0}^{m-2} a_{k} \int_{\partial B(x \rho)} \Delta^{2}(\nu-2 i I)^{k} u(y) d S(y) .
\end{aligned}
$$

Integrating both sides with respect to $\rho$ over the interval $(0, r)$, we obtain 


$$
\begin{aligned}
\Delta^{k} u(x) & =r^{-n} \sum_{k=0}^{m-2} a_{k}^{\prime} \int_{B(x, r)} \Delta^{2}(\nu-2 i I)^{k} u(y) d y \\
& =r^{-n-1} \sum_{k=0}^{m-2} a_{k}^{\prime} \int_{\partial B(x, r)} \nu \Delta^{\imath-1}(\nu-2 i I)^{k} u(y) d S(y) \\
& =r^{-n-1} \sum_{k=0}^{m-2} a_{k}^{\prime} \int_{\partial B(x, r)} \Delta^{\imath-1}(\nu-2(\imath-1) I)(\nu-2 i I)^{k} u(y) d S(y) .
\end{aligned}
$$

Repeating this process, we finally obtain

$$
\Delta^{\imath} u(x)=r^{-n-2 \imath} \sum_{k=0}^{m-\imath} a_{k}^{\prime \prime} \int_{B(x, r)} \nu(\nu-2 I) \cdots(\nu-2(\imath-1) I)(\nu-2 i I)^{k} u(y) d y,
$$

which is of the form (3).

The following fact can be proved easily (cf. [6; Lemma 5]).

LEMMA 3. Let $u$ be a function in $C^{1}\left(R_{+}^{n}\right)$ such that

$$
\int_{G}\left|\nabla_{1} u(x)\right|^{p} x_{n}^{a} d x<\infty, \quad p>1,
$$

for any bounded open set $G$ in $R_{+}^{n}$. Then

$$
\int_{G}|u(x)|^{p} x_{n}^{\beta} d x<\infty
$$

for any bounded open set $G$ in $R_{+}^{n}$, where $\beta=\alpha-p$ if $\alpha>p-1$ and $\beta>-1$ if $\alpha=p-1$.

By $[6$; Lemma 4$]$ we have

LEMMA 4. Let $k$ be a positive integer, $p>1$ and $\beta<p-1$. Let $u$ be a functzon in $C^{k}\left(R_{+}^{n}\right)$ such that

$$
\int_{G}\left|\nabla_{k} u(x)\right|^{p} x_{n}^{\beta} d x<\infty
$$

for any bounded open set $G$ in $R_{+}^{n}$. If we set

$$
A=\left\{\xi \in \partial R_{+}^{n} ; \int_{B(\xi, 1) \cap R_{+}^{n}}|\xi-x|^{k-n}\left|\nabla_{k} u(y)\right| d y=\infty\right\},
$$

then $B_{k-\beta / p, p}(A)=0$.

LEMMA 5. Let $f$ be a nonnegative measurable function on $R_{+}^{n}$ such that $\int_{G} f(y) d y<\infty$ for any bounded open set $G$ in $R_{+}^{n}$, and define

$$
B_{\delta}=\left\{\xi \in \partial R_{+}^{n} ; \int_{B(\xi, 1) \cap R_{+}^{n}}\left(\left|\xi^{\prime}-y^{\prime}\right|^{2 \gamma}+y_{n}^{2}\right)^{-(l+\delta) / 2} f(y) y_{n}^{\delta} d y=\infty\right\},
$$


where $l \geqq 0$ and $\gamma \geqq 1$. Then $H_{\gamma l}\left(B_{\delta}\right)=0$ for any $\delta>0$ : in case $l=0$, thrs implies that $B_{\delta}$ is empty.

Proof. Suppose $H_{\gamma l}\left(B_{\delta}\right)>0$. Then by $[1$; Theorems 1 and 3 in $\S$ II] we can find a nonnegative measure $\mu$ with compact support in $\partial R_{+}^{n}$ such that $\mu\left(B_{\delta}\right)>0$ and

$$
\mu(B(x, r)) \leqq r^{r l} \quad \text { for any } x \text { and } r .
$$

Then $\int\left(\left|\xi^{\prime}-y^{\prime}\right|^{2 \gamma}+y_{n}^{2}\right)^{-(l+\delta) / 2} d \mu(\xi) \leqq$ const. $y_{n}^{-\delta}$ for $y \in R_{+}^{n}$. Hence

$$
\begin{aligned}
\infty & =\int\left\{\int_{B(\xi, 1) \cap R_{+}^{n}}\left(\left|\xi^{\prime}-y^{\prime}\right|^{2 \gamma}+y_{n}^{2}\right)^{-(l+\delta) / 2} f(y) y_{n}^{\delta} d y\right\} d \mu(\xi) \\
& \leqq \int_{G}\left\{\int\left(\left|\xi^{\prime}-y^{\prime}\right|^{2 \gamma}+y_{n}^{2}\right)^{-(l+\delta) / 2} d \mu(\xi)\right\} f(y) y_{n}^{\delta} d y \\
& \leqq \text { const. } \int_{G} f(y) d y<\infty,
\end{aligned}
$$

which is a contradiction. Here $G=\underset{\xi \in \text { supp } \mu}{\cup} B(\xi, 1) \cap R_{+}^{n}$.

LemMa 6. Let $k$ be a positive integer, $p>1$ and $\beta<p-1$. Let $K$ be a Borel measurable function on $R^{n}$ such that $\left|\nabla_{l} K(x)\right| \leqq|x|^{k-l-n}$ on $R^{n}-\{0\}$ for $l=0,1$. $\cdots, k-1$, and define

$$
u(x)=\int K(x-y) f(y) d y
$$

for a nonnegative measurable function $f$ on $R^{n}$ such that $\int|x-y|^{k-n} f(y) d y \neq \infty$ and $\int_{G} f(y)^{p}\left|y_{n}\right|^{\beta} d y<\infty$ for any bounded open set $G \subset R^{n}$. Set

$$
E_{l, \gamma}=\left\{\xi \in \partial R_{+}^{n} ; \lim _{x \rightarrow \xi, x \in T_{\gamma}(\xi, a)} \int_{B\left(x, x_{n} / 2\right)}\left|\nabla_{l} u(y)\right|^{p} y_{n}^{l p-n} d y>0 \text { for some } a>0\right\}
$$

for $\gamma \geqq 1$ and $l=1, \cdots, k-1$. Then $H_{\gamma(n-k p+\beta)}\left(E_{l, \gamma}\right)=0$ if $n-k p+\beta>0$, and $E_{l, \gamma}$ is empty if $n-k p+\beta \leqq 0$.

Proof. Define

$$
E_{\gamma}=\left\{\xi \in \partial R_{+}^{n} ; \lim _{r \downarrow 0} \sup r^{\gamma(k p-\beta-n)} \int_{B(\xi, r)} f(y)^{p}\left|y_{n}\right|^{\beta} d y>0\right\}
$$

for $\gamma \geqq 1$. Then, in view of $[7$; Lemma 2$]$, we see that $H_{\gamma(n-k p+\beta)}\left(E_{\gamma}\right)=0$ if $n-k p+\beta>0$ and $E_{\gamma}$ is empty if $n-k p+\beta \leqq 0$.

Let $l$ be a positive integer such that $l<k$. Then for almost every $x$,

$$
\left|\nabla_{\iota} u(x)\right| \leqq \int|x-y|^{k-l-n} f(y) d y=U_{1}(x)+U_{2}(x)+U_{3}(x),
$$

where 


$$
\begin{aligned}
& U_{1}(x)=\int_{B\left(x, c x_{n}\right)}|x-y|^{k-l-n} f(y) d y, \quad 0<c<1 / 3, \\
& U_{2}(x)=\int_{B(\xi, 2|x-\xi|)-B\left(x, c x_{n}\right)}|x-y|^{k-t-n} f(y) d y, \\
& U_{3}(x)=\int_{R^{n}-B(\xi, 2|x-\xi|)}|x-y|^{k-l-n} f(y) d y .
\end{aligned}
$$

We first note from Hölder's inequality that

$$
\lim _{r \downarrow 0} r^{k-n} \int_{B(\xi, r)} f(y) d y=0
$$

if $\xi \in \partial R_{+}^{n}-E_{1}$ and hence if $\xi \in \partial R_{+}^{n}-E_{\gamma}$. Setting $\varepsilon(\eta)=\sup _{0<r \leqq \eta} r^{k-n} \int_{B(\xi, r)} f(y) d y$ for $\eta>0$, we have

$$
\begin{aligned}
U_{3}(x) & \leqq \text { const. } \int_{R^{n}-B(\xi, 2|x-\xi|)}|y-\xi|^{k-l-n} f(y) d y \\
& \leqq \text { const. }\left\{\int_{R^{n}-B(\xi, \eta)}|y-\xi|^{k-l-n} f(y) d y+\varepsilon(\eta)|x-\xi|^{-\ell}\right\} .
\end{aligned}
$$

Consequently, $\lim _{z \rightarrow \xi, z \in R_{+}^{n}} \int_{B\left(z, z_{n} / 2\right)} U_{3}(x)^{p} x_{n}^{l p-n} d x \leqq$ const. $\varepsilon(\eta)^{p}$. This implies that

$$
\lim _{z \rightarrow \xi, z \in R_{+}^{n}} \int_{B(z, z n / 2)} U_{3}(x)^{p} x_{n}^{l p-n} d x=0 .
$$

By Hölder's inequality,

so that

$$
U_{1}(x) \leqq \text { const. } x_{n}^{(k-l) / p^{\prime}}\left\{\int_{B\left(x, c x_{n}\right)}|x-y|^{k-l-n} f(y)^{p} d y\right\}^{1 / p},
$$

$$
\begin{aligned}
& \int_{B\left(z, z_{n} / 2\right)} U_{1}(x)^{p} x_{n}^{l p-n} d x \\
& \leqq \text { const. } z_{n}^{(k-l) p / p^{\prime}+l p-n} \int_{B\left(z,(1+3 c) z_{n} / 2\right)} f(y)^{p}\left\{\int_{B\left(z, z_{n} / 2\right)}|x-y|^{k-l-n} d x\right\} d y \\
& \leqq \text { const. } z_{n}^{k p-\beta-n} \int_{B(\xi, 2|z-\xi|)} f(y)^{p}\left|y_{n}\right|^{\beta} d y .
\end{aligned}
$$

Therefore if $n-k p+\beta>0$ and $\xi \in \partial R_{+}^{n}-E_{\gamma}$, then

$$
\lim _{z \rightarrow \xi, z \in T_{\gamma}^{(\xi, a)}} \int_{B\left(z, z_{n} / 2\right)} U_{1}(x)^{p} x_{n}^{l p-n} d x=0 ;
$$

if $n-k p+\beta \leqq 0$, then

$$
\lim _{z \rightarrow \xi, z \in R_{+}^{n}} \int_{B\left(z, z_{n} / 2\right)} U_{1}(x)^{p} x_{n}^{\iota p-n} d x=0 .
$$


Letting $\eta=2|x-\xi|$ and $M=\int_{B(\xi, \eta)} f(y)^{p}\left|y_{n}\right|^{\beta} d y$, we have by [7; Lemma 5],

$$
U_{2}(x)^{p} \leqq \text { const. }\left\{\begin{array}{lll}
x_{n}^{(k-l) p-\beta-n} M & \text { if } & (k-l) p-\beta-n<0, \\
{\left[\log \left(\eta x_{n}^{-1}+2\right)\right]^{p-1} M} & \text { if } \quad(k-l) p-\beta-n=0, \\
\eta^{(k-l) p-\beta-n} M & \text { if } \quad(k-l) p-\beta-n>0 .
\end{array}\right.
$$

If $z \in T_{\gamma}(\xi, a) \cap B(\xi, 1)$ and $x \in B\left(z, z_{n} / 2\right)$, then there exists $a^{\prime}>0$ such that $x \in T_{\gamma}\left(\xi, a^{\prime}\right)$. Hence we obtain

$$
\int_{B\left(z, z_{n} / 2\right)} U_{2}(x)^{p} x_{n}^{l p-n} d x \leqq \text { const. } \begin{cases}z_{n}^{k p-\beta-n} M & \text { if } \quad(k-l) p-\beta-n<0, \\ z_{n}^{l p}\left[\log \left(\eta z_{n}^{-1}+2\right)\right]^{p-1} M & \text { if } \quad(k-l) p-\beta-n=0, \\ z_{n}^{l p} \eta^{(k-l) p-\beta-n} M & \text { if } \quad(k-l) p-\beta-n>0,\end{cases}
$$

which tends to zero as $z \rightarrow \xi, z \in T_{\gamma}(\xi, a)$, if $k p-\beta-n<0$ and $\xi \notin E_{\gamma}$, and as $z \rightarrow \xi$ if $k p-\beta-n \geqq 0$. Thus we proved that $E_{l, \gamma} \subset E_{\gamma}$ if $n-k p+\beta>0$ and $E_{l, \gamma}$ is empty if $n-k p+\beta \leqq 0$. The proof is now complete.

COROLlary. Let $k, p$ and $\beta$ be as in the lemma. Let $u$ be a function in $C^{k}\left(R_{+}^{n}\right)$ such that $\int_{G}\left|\nabla_{k} u(x)\right|^{p} x_{n}^{\beta} d x<\infty$ for any bounded open set $G$ in $R_{+}^{n}$, and define $E_{l, \gamma}$ as in the lemma. Then $H_{\gamma(n-k p+\beta)}\left(E_{l, \gamma}\right)=0$ if $n-k p+\beta>0$ and $E_{l, \gamma}$ is empty if $n-k p+\beta \leqq 0$.

Proof. Let $q=p$ if $\beta \leqq 0$ and $1<q<p /(\beta+1)$ if $\beta>0$. By Hölder's inequality we have

$$
\int_{G}\left|\nabla_{k} u(x)\right|^{q} d x<\infty
$$

for any bounded open set $G$ in $R_{+}^{n}$. By Theorem 5 and its proof in [10; Chap. VI], we can find a function $v \in L_{\mathrm{loc}}^{q}\left(R^{n}\right)$ such that $v=u$ a.e. on $R_{+}^{n}$,

$$
\int_{G}\left|\nabla_{k} v(x)\right|^{q} d x<\infty \text { and } \int_{G}\left|\nabla_{k} v(x)\right|^{p}\left|x_{n}\right|^{\beta} d x<\infty
$$

for any bounded open set $G$ in $R^{n}$, where the derivatives are taken in the sense of distributions.

We shall show that $H_{\gamma(n-k p+\beta)}\left(E_{l, \gamma} \cap B(0, r)\right)=0$ if $n-k p+\beta>0$ and $E_{l, \gamma} \cap$ $B(0, r)$ is empty if $n-k p+\beta \leqq 0$ for any $r>0$. Let $r>0$ be fixed, and take a function $\phi \in C_{0}^{\infty}\left(R^{n}\right)$ such that $\phi=1$ on $B(0,2 r)$. Set $w=\phi v$. Then by [5; Theorem 4.1],

$$
w(x)=\sum_{|\lambda|=k} a_{\lambda} \int \frac{(x-y)^{\lambda}}{|x-y|^{n}} D^{\lambda} w(y) d y \quad \text { a.e. on } R^{n} .
$$

Since $w$ is considered to be continuously $k$ times differentiable on $R_{+}^{n}$, the right hand side is also continuously $k$ times differentiable on $R_{+}^{n}$ and the equality is 
considered to hold at every point of $R_{+}^{n}$. Further,

$$
\int_{B(0, r)}\left|\nabla_{k} w(y)\right|^{p}\left|y_{n}\right|^{\beta} d y<\infty .
$$

Thus the proof of Lemma 6 shows that $H_{\gamma(n-k p+\beta)}\left(E_{l, r} \cap B(0, r)\right)=0$ if $n-k p+\beta$ $>0$ and $E_{l, r} \cap B(0, r)$ is empty if $n-k p+\beta \leqq 0$. By noting the arbitrariness of $r$, we conclude the proof.

\section{Proof of the theorem.}

Let $u$ be as in the theorem. If $\alpha<p-1$, we let $k=1$, and if $\alpha \geqq p-1$, then we let $k$ be a positive integer such that $(k-1) p-1<\alpha<k p-1$. Define $\beta=\alpha-$ $(k-1) p$. Then $\beta<p-1$, and, in view of Lemma 3 ,

$$
\int_{G}\left|\nabla_{m-l} u(x)\right|^{p} x_{n}^{\alpha-l p} d x<\infty
$$

for any bounded open set $G$ in $R_{+}^{n}$ and $l=0,1, \cdots, k-1$.

Let $q=p$ if $\beta \leqq 0$ and $1<q<p /(\beta+1)$ if $\beta>0$. By Hölder's inequality we have

$$
\int_{G}\left|\nabla_{m-k+1} u(x)\right|^{q} d x<\infty
$$

for any bounded open set $G$ in $R_{+}^{n}$. As in the proof of the corollary to Lemma 6 , we can find a function $v \in L_{\mathrm{loc}}^{q}\left(R^{n}\right)$ such that $v=u$ a.e. on $R_{+}^{n}$,

$$
\int_{G}\left|\nabla_{m-k+1} v(x)\right|^{q} d x<\infty
$$

and

$$
\int_{G}\left|\nabla_{m-k+1} v(x)\right|^{p}\left|x_{n}\right|^{\beta} d x<\infty
$$

for any bounded open set $G$ in $R^{n}$.

Define

$$
\begin{aligned}
A & =\left\{\xi \in \partial R_{+}^{n} ; \int_{B(\xi, 1)}|\xi-y|^{m-k+1-n}\left|\nabla_{m-k+1} v(y)\right| d y=\infty\right\}, \\
E_{l, \gamma} & =\left\{\xi \in \partial R_{+}^{n} ; \lim _{x \rightarrow \xi, x \in T_{\gamma} \sup _{\gamma}(\xi)} \int_{B\left(x, x_{n} / 2\right)}\left|\nabla_{l} u(y)\right|^{p} y_{n^{l}}^{l p-n} d y>0 \text { for some } a>0\right\}, \\
F_{\eta} & =\left\{\xi \in \partial R_{+}^{n} ; \lim _{r \downarrow 0} \sup r^{-\eta} \int_{B(\xi, r)}\left|\nabla_{m-k+1} v(y)\right|^{p}\left|y_{n}\right|^{\beta} d y>0\right\} \quad \text { for } \eta>0, \\
F_{0} & =\left\{\xi \in \partial R_{+}^{n} ; \lim _{r \downarrow 0} \sup \left(\log r^{-1}\right)^{p-1} \int_{B(\xi, r)}\left|\nabla_{m-k+1} v(y)\right|^{p}\left|y_{n}\right|^{\beta} d y>0\right\}
\end{aligned}
$$

and

$$
E_{\gamma}=A \cup\left(\bigcup_{l=1}^{m} E_{l, r}\right) \cup F_{\gamma(n-m p+\alpha)} \quad \text { for } n-m p+\alpha \geqq 0
$$


We shall show below that $\lim _{x \rightarrow \xi, x \in T_{\gamma}(\xi, a)} u(x)$ exists and is finite for any $\xi \in \partial R_{+}^{n}-E_{\gamma}$ and any $a>0$; in case $n-m p+\alpha<0$, our proof below shows that $u(x)$ has a finite limit as $x \rightarrow \xi, x \in R_{+}^{n}$, for any $\xi \in \partial R_{+}^{n}$.

By Lemma $4, B_{m-\alpha / p, p}(A)=0$. In view of Lemma 5 ,

$$
\int_{T_{\gamma}(\xi, a) \cap B(\xi, 1)}\left|\nabla_{l} u(x)\right|^{p} x_{n}^{l p-n} d x<\infty, \quad l=m-k+1, \cdots, m,
$$

for any $a>0$ and any $\xi \in \partial R_{+}^{n}$ except for a set $B_{\gamma}$ such that $H_{\gamma(n-m p+\alpha)}\left(B_{\gamma}\right)=0$ if $n-m p+\alpha>0$ and $B_{r}$ is empty if $n-m p+\alpha \leqq 0$, so that

$$
H_{\gamma(n-m p+\alpha)}\left(E_{l, \gamma}\right)=0 \quad \text { if } \quad n-m p+\alpha \geqq 0 \text { and } l=m-k+1, \cdots, m \text {. }
$$

The corollary to Lemma 6 implies that $H_{\gamma(n-m p+\alpha)}\left(E_{l, \gamma}\right)=0$ if $n-m p+\alpha>0$ and $l=1, \cdots, m-k$, and $E_{l, \gamma}$ is empty if $n-m p+\alpha \leqq 0$ and $l=1, \cdots, m-k$. Thus, with the aid of $[7$; Lemmas 2 and 3$]$, we see that $H_{\gamma(n-m p+\alpha)}\left(E_{\gamma}\right)=0$ if $n-m p+\alpha>0$, and $B_{n / p, p}\left(E_{\infty}\right)=0$ if $n-m p+\alpha=0$, where $E_{\infty} \equiv \bigcup_{\gamma>1} E_{\gamma}=A \cup F_{0}$.

Let $\xi \in \partial R_{+}^{n}-E_{\gamma}$, and take a function $\phi \in C_{0}^{\infty}\left(R^{n}\right)$ such that $\phi=1$ on $B(\xi, 2)$. Write $m-k+1=2 s+s^{*}$, where $s$ and $s^{*}$ are nonnegative integers such that $0 \leqq s^{*} \leqq 1$. Setting $w=\phi v$, we have the following integral representation (cf. [5; Theorems 4.1 and 4.2$]$ ):

$$
w(x)=U(x ; w) \equiv \begin{cases}\int K_{2 s}(x-y) \Delta^{s} w(y) d y & \text { if } s^{*}=0, \\ \sum_{j=1}^{n} \int \frac{\partial K_{2 s+2}}{\partial x_{\jmath}}(x-y)\left(\frac{\partial}{\partial y_{\jmath}} \Delta^{s} w(y)\right) d y & \text { if } s^{*}=1,\end{cases}
$$

holds for almost every $x \in R^{n}$, where $K_{2 l}(x)=C_{l}|x|^{2 l-n}$ if $2 l<n$ or $n$ is odd, and $K_{2 l}(x)=C_{l}|x|^{2 l-n} \log |x|$ if $2 l \geqq n$ and $n$ is even; the constants $C_{l}$ are chosen so that $U(x ; \phi)=\psi$ for any $\phi \in C_{0}^{\infty}\left(R^{n}\right)$. Since $w$ is infinitely differentiable on $R_{+}^{n}, U(x ; w)$ is continuous on $R_{+}^{n}$ and $w(x)=U(x ; w)$ holds for any $x \in R_{+}^{n}$.

We shall prove the theorem only in the case $s^{*}=1$; the case $s^{*}=0$ can be proved similarly. Write $U(x ; w)=U_{1}(x)+U_{2}(x)+U_{3}(x)$, where

$$
\begin{aligned}
& U_{1}(x)=\sum_{\jmath=1}^{n} \int_{B\left(x, x_{n} / 2\right)} \frac{\partial K_{2 s+2}}{\partial x_{\jmath}}(x-y)\left(\frac{\partial}{\partial y_{\jmath}} \Delta^{s} w(y)\right) d y, \\
& U_{2}(x)=\sum_{\jmath=1}^{n} \int_{B(x,|x-\xi| / 2)-B\left(x, x_{n} / 2\right)} \frac{\partial K_{2 s+2}}{\partial x_{\jmath}}(x-y)\left(\frac{\partial}{\partial y_{\jmath}} \Delta^{s} w(y)\right) d y, \\
& U_{3}(x)=\sum_{j=1}^{n} \int_{R^{n}-B(x,|x-\xi| / 2)} \frac{\partial K_{2 s+2}}{\partial x_{\jmath}}(x-y)\left(\frac{\partial}{\partial y_{\jmath}} \Delta^{s} w(y)\right) d y .
\end{aligned}
$$

Since $\xi \oplus A$ by our assumption, $\int\left|\nabla_{1} K_{2 s+2}(\xi-y)\right|\left|\nabla_{2 s+1} w(y)\right| d y<\infty$, so that Lebesgue's dominated convergence theorem implies that $\lim U_{3}(x)$ exists and is finite as $x \rightarrow \xi, x \in R_{+}^{n}$. 
Define $W(x)=\int_{B(\xi, 2|x-\xi|)}\left|\nabla_{2 s+1} w(y)\right|^{p}\left|y_{n}\right|^{\beta} d y$. As in [7; Lemma 5], we have

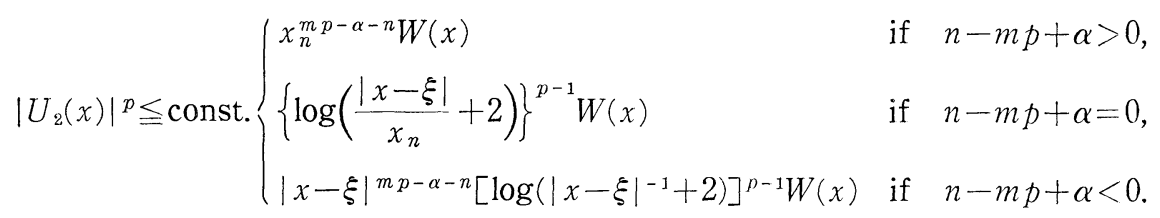

Since $w(x)=v(x)$ on $B(\xi, 1), \lim _{x \rightarrow \xi, x \in T_{\gamma}(\xi, a)} U_{2}(x)=0$ for any $a>0$.

Set $k_{s}(r)=K_{2 s+2}(x)$, where $r=|x|$. If $n-m p+\alpha \geqq 0$, then $2 s+1=m-k+1<n$. First suppose $2 s+2 \leqq n$. Then

$$
\begin{aligned}
U_{1}(x)= & -\sum_{j=1}^{n} k_{s}\left(x_{n} / 2\right) \int_{\partial B\left(x, x_{n} / 2\right)} \frac{\partial}{\partial y_{j}} \Delta^{s} u(y) \frac{y_{j}-x_{j}}{|y-x|} d S(y) \\
& +\int_{B\left(x, x_{n} / 2\right)} K_{2 s+2}(x-y) \Delta^{s+1} u(y) d y \\
= & -\int_{B\left(x, x_{n} / 2\right)}\left\{k_{s}\left(x_{n} / 2\right)-K_{2 s+2}(x-y)\right\} \Delta^{s+1} u(y) d y \\
= & -\int_{0}^{x_{n}^{\prime \prime 2}}\left\{k_{s}\left(x_{n} / 2\right)-k_{s}(r)\right\}\left\{\int_{\partial B(x, r)} \Delta^{s+1} u(y) d S(y)\right\} d r \\
= & -\sum_{i=1}^{m-s} c_{\imath} \Delta^{\imath+s} u(x) \int_{0}^{x_{n} / 2}\left\{k_{s}\left(x_{n} / 2\right)-k_{s}(r)\right\} r^{n-1+22-2} d r \\
= & -\sum_{i=1}^{m-s} c_{i}^{\prime} \Delta^{2+s} u(x) x_{n}^{22+2 s} \\
= & x_{n}^{-n} \sum_{0<i \lambda \mid \leq m} c_{\lambda} \int_{B\left(x, x_{n} / 2\right)}(y-x)^{\lambda} D^{\lambda} u(y) d y
\end{aligned}
$$

by Lemmas 1 and 2, where $x \in B(\xi, 1) \cap R_{+}^{n}$, so that $u(x)=w(x)$ there. Hence it follows from Hölder's inequality that

$$
\left|U_{1}(x)\right| \leqq \text { const. } \sum_{l=1}^{m}\left(\int_{B\left(x, x_{n} / 2\right)}\left|\nabla_{l} u(y)\right|^{p} y_{n}^{l p-n} d y\right)^{1 / p},
$$

which tends to zero as $x \rightarrow \xi, x \in T_{\gamma}(\xi, a)$, since $\xi \notin \bigcup_{l=1}^{m} E_{l, \gamma}$. Thus the proof of the theorem is complete.

\section{Further results and remarks.}

Let $D$ be a special Lipschitz domain as defined in Stein [10; Chap. V1]. Then similar results can be shown to hold for $u$ which is polyharmonic of order $m+1$ in $D$ and satisfies 


$$
\int_{D}\left|\nabla_{m} u(x)\right|^{p} d(x)^{\alpha} d x<\infty, \quad p>1, \alpha<m p-1,
$$

if we replace $T_{\gamma}(\xi, a)$ by the set $\left\{x \in D ;|x-\xi|<a d(x)^{1 / \gamma}\right\}$. Here $d(x)$ denotes the distance from $x$ to the boundary $\partial D$.

Finally we give an open problem: If $u$ is a function which is polyharmonic of order $m+1$ in $R_{+}^{n}$ and satisfies (1) with $p>1$ and $\alpha=m p-1$ for any bounded open set $G$ in $R_{+}^{n}$, then does there exist a set $E$ such that $H_{n-1}(E)=0$ and $u$ has a nontangential limit at any $\xi \in \partial R_{+}^{n}-E$ ? By a well known result $[10$; Theorem 4 in Chap. VII], this is true for a harmonic function $u$ in $R_{+}^{n}$ satisfying (1) with $1<p \leqq 2$ and $\alpha=p-1$ for any bounded open set $G$ in $R_{+}^{n}$. In view of the proofs of [8; Theorem 1] and our theorem, we have the following result: If $u$ is a function which is polyharmonic of order $m+1$ in $R_{+}^{n}$ and satisfies (1) with $p>1$ and $\alpha=m p-1$ for any bounded open set $G$ in $R_{+}^{n}$, then there exists a. set $E \subset \partial R_{+}^{n}$ such that $H_{n-1}(E)=0$ and

$$
C\left(\xi ; u, l_{\xi}\right)=C\left(\xi ; u, T_{1}(\xi, a)\right)
$$

for any $a>0$ and any $\xi \in \partial R_{+}^{n}-E$, where $\left.C(\xi ; u, F)=\bigcap_{r>0} \overline{u(F \cap B(\xi, r)}\right)$ for a set $F \subset R_{+}^{n}$ and $l_{\xi}=\{\xi+(0, \cdots, 0, t) ; t>0\}$.

\section{REFERENCES}

[1] L. Carleson, Selected problems on exceptional sets, Van Nostrand, Princeton, 1967.

[2] A.B. Cruzeiro, Convergence au bord pour les fonctions harmoniques dans $R^{d}$ de la classe de Sobolev $W_{1}^{a}$, C. R. Acad. Sci., Paris 294 (1982), 71-74.

[3] J. EDENHOFER, Integraldarstellung einer $m$-polyharmonischen Funktion, deren Funktionswerte und erste $m-1$ Normalableitungen auf einer Hyperspäre gegeben sind, Math. Nachr., 68 (1975), 105-113.

[4] N.G. Meyers, A theory of capacities for potentials in Lebesgue classes, Math. Scand., 26 (1970), 255-292.

[5] Y. MizutA, Integral representations of Beppo Levi functions of higher order, Hiroshima Math. J., 4 (1974), 375-396.

[6] Y. Mizuta, Existence of varıous boundary limits of Beppo Levi functions of higher order, Hiroshima Math. J., 9 (1979), 717-745.

[7] Y. Mizuta, On the behavior of potentials near a hyperplane, Hiroshıma Math. J., 13 (1983), 529-542.

[8] Y. Mizuta And B.H. Qui, On the existence of non-tangential limits of polyharmonic functions, Hiroshima Math. J., 8 (1978), 409-414.

[9] M. M. Nicolesco, Recherches sur les fonctions polyharmoniques, Ann. Sci. École Norm. Sup., 52 (1935), 183-220.

[10] E. M. Stein, Singular integrals and differentiability propertıes of functions, Prince. ton Univ. Press, Princeton, 1970.

Department of Mathematics

Faculty of Integrated Arts and Sciences

Hiroshima Universiny

Hiroshima 730, JAPAN 